BIO Web of Conferences 1, 00053 (2011)

DOI: $10.1051 /$ bioconf $/ 20110100053$

(C) Owned by the authors, published by EDP Sciences, 2011

\title{
Evaluation of the light weight Juggling system
}

\author{
Julien Lagarde*, Gregory Zelic*, Carlo Alberto Avizzano ${ }^{* \dagger}$, Vittorio Lippi ${ }^{* \dagger}$, Emanuelle \\ Ruffaldi $^{*}$, Hagar Zalmanov ${ }^{\ddagger}$, Rotem Lammfromm ${ }^{\ddagger}$, Vered Erev-Yehene ${ }^{\ddagger}$, Nick Gillian ${ }^{\dagger}$, Sile \\ O’Modrain ${ }^{\dagger}$, Denis Mottet*, Danny Gopher \\ (*) University Montpellier 1, Montpellier, France \\ $\left(^{*}\right)$ Scuola Superiore S. Anna, Pisa, Italy \\ (*) TECHNION, Haifa, Israel \\ (\$) Queen's University Belfast, Belfast, Northern Ireland \\ E-mail: julien.lagarde@univ-montp1.fr
}

\begin{abstract}
This paper presents the training of juggling skills, a highly complex problem of coordination, under tight time and space constraints. This training is achieved with a simple training platform, a light weight juggling platform (LWJ), and is compared to training with real balls. The principle directing the design of the platform is to obtain a simple tool. The simplification, in comparison to real world juggling, is based on the identification of invariant properties in the spatiotemporal coordination of intermediate and expert jugglers. Two classes training solutions were added to the LWJ: An audio-tactile pacing or augmented multimodal environment, and the manipulation of cognitive components of the juggling skills. The transfer to juggling with real balls was evaluated in four different experiments.
\end{abstract}

\section{Introduction}

The science of juggling has brought significant insights for the understanding of complex cognitive and perceptivo-motor skills. Juggling represents an emblematic case of goal directed behavior under severe physical constraints, and as such has attracted attention in several fields, like mathematics, motor control, and neuroscience (Beek and Lewbel, 1995; Cardinal et al., 2006; Draganski et al., 2004; Driemeyer et al., 2008). Even in one of these easiest trick, the three balls cascade (se Figure 1), the acquisition of juggling skills requires a large amount of practice (Huys et al., 2004), imposes clearly drastic changes in the repertoire of coordination between vision and arm movements (Beek and van Santvoord, 1992; Draganski et al., 2004; Huys et al., 2004), and is characterized by a high degree of spatio-temporal behavioral organization
(Post et al., 2000). This pattern of whole body movements is essentially captured by relative timing, readily measured by frequency and phase relations between hands, arms, eyes, and postural movements (Huys et al., 2004).

The basic design of the LWJ and the accompanying training strategies were driven by these findings. Accordingly we focused on the invariant relative timing pervasive in juggling, at the expense of other properties present in the real environment, notably kinetic rendering of the interaction with the balls. We assumed that this choice would maximize the transfer of training to juggling with real balls while minimizing the technological cost of the development of the training platform.

\section{Integrated Technology}

\section{Movement tracking}

Pohlemus is a 3D tracker. It is used to get the hand position in real time. It works getting the position and the orientation (not used in this implementation) of two sensors hold by the user.

The application is controlled by the user just through this device: ball tossing is triggered by hand acceleration and ball speed just after the toss is set to the hand speed.

Hand speed and acceleration are computed from position by successive numeric derivative. This means that disturbances are very critical in this application. Polhemus works using electromagnetic fields: this makes it very sensible to electromagnetic disturbance. Such devices like vibrating motors should not be too close to the sensors. Problems can arise also near metallic objects (the cave we have at PERCRO is hence non suitable to be used, being it supported by a metallic structure) or near the monitor.

This is an Open Access article distributed under the terms of the Creative Commons Attribution-Noncommercial License 3.0, which permits unrestricted use, distribution, and reproduction in any noncommercial medium, provided the original work is properly cited. 


\section{The Datakit Simulink Library}

The library allows to set up the data storage for experiment sessions and data retrieval for further analysis: Database, entities and attributes are represented by Simulink blocks; hierarchies between entities are specified giving the parent id as input to children blocks; data to be stored are given as input; retrieved data are given as block output; the same block can be used in READ or WRITE mode .Blocks are implemented as instances of a cpp S-function, function parameters can be inserted through masks associated to blocks opened with a double click.

In terms of storage system there are already some advantages in this solution: first, data are saved in a structured form that can be specified by connecting simulink blocks representing 'entities' and datablock according to hierarchical relationship (entities are nodes of a three) then the storage function can use parallel threads to optimize the performance. This solution has not been released so far to make easier to patners that have not yet used Datakit to perform experiments and return a feedback about the system.

\section{Training solutions to accelerate learning}

\section{Audio-tactile training}

The first training accelerator proposed is based on the results of effective stabilization obtained for various examples of interlimb and limb- environment coordination dynamics [22,23]. Juggling skills is characterized by the assembly of multiple subskills, including coordination with multifrequency relations, mainly among the eyes looking at the ball pattern in the air and the hand [15]. Our accelerator is a multifrequency audio-tactile sensory stimulation, tactile vibrations were provided in antiphase at the wrists. The period of the sound beeps corresponded to one ball cycle, and the period of the tactile stimuli to the period of the hand. We compared to a control group, including only training to juggle with real balls, two audio-tactile LWJ groups: One with an open loop mode with preprogrammed timing of sound and vibration, and one with a closed loop mode in which the sound and vibration provided at times of throws. We recruited fifteen adults from Montpellier University 1 having no prior juggling experiment which were paid for their participation. The participants practiced the three balls cascade during two weeks for 10 sessions including pretest and nine training sessions, and then passed a posttest session one week later. Five participants were assigned to each of the groups and performed nine learning session. The control group consisted in training with real balls. Each session lasted 20 minutes without supplementary instructions. Each session finished with the five minutes test during which participants have to perform as many consecutive catches as possible and the number of consecutive catches was recorded. The evaluation of the level of transfer of learning done during this session with real balls, participants were instructed to juggle as many consecutive catches as possible and the number of consecutive catches was recorded.

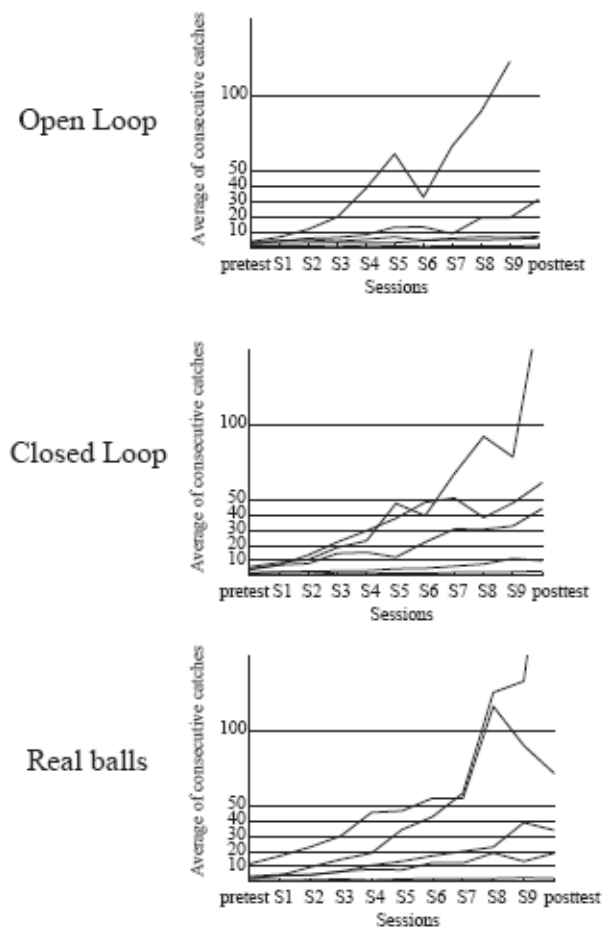

Figure 1. Comparison of juggling performance with real balls between the three groups of training. The average number of consecutive catches across trials is shown, for the five subjects of each group, recorded during the pre- and post- tests, and at the end of each of the nine training sessions.

The transfer of training to real balls was more efficient in the Open loop condition than in the Closed Loop condition (Figure 1). Like in the Control group, four subjects in the Open Loop condition reached a performance of at least ten consecutive catches, while in the Closed Loop condition only one subject reached this level.

\section{Ball's speed manipulation}

A key requirement in the ability to juggle a specific trick and distinguishing it from others is the representation and memorization of the required 
sequential pattern of tossing and catching the juggled objects.

The task speed manipulation accelerator focuses on the manipulation of the overall juggling task cycle speed. Learning a new skill (the ability to achieve efficiently a desired outcome/goal), is commonly described in human performance theories $[1,11,19]$ as a continuous process of moving from a slow controlled modes of processing and response to rapid efficient "automatic" performance. The virtual environment of the LWJ provides the opportunity for a general slow down of the overall time clock (pace) of the task, allowing trainees to observe, perform and practice the task in "slow motion", while all relationships between elements, the pattern or the order in which they have respond to stays intact.

In the second experiment Students with no prior juggling experience trained in the LWJ on 3 ball cascade juggling for 8 sessions, each comprising 10 four minute practice trials, under task speed change. There were 6 different speeds, one was the normal, 3 were slower and two faster than normal. Practice started at slow speed and trainees graduated to faster speed levels when meeting the required graduation criterion of 2 consecutive juggling cycles within a 4 minute practice trial. Training starting from slow speed was expected to allow subjects to develop a better representation of the trick pattern. Juggling performance of the speed change group was compared on a 9th test session performed at normal speed to a control group which practiced for 8 sessions only in normal speed. Performance was measured by the average representing the longest sequence of balls catch and toss without a fall within a 4 minute block. An additional 10th session was given when both experimental and control group were required to perform two new tricks reversed cascade and snake.

The results show that the group trained with speed changes obtained significantly higher juggling scores than the control group on the 9th test session (14.86 vs. 8.75 respectively). In addition, subjects trained under speed change obtained significantly higher scores when transferred to the reversed cascade trick which is a variant of original trick they were trained on $(7.00 \mathrm{vs}$ 3.67 respectively). No difference, were observed on the transfer to the snake trick which is very different and much more difficult.

\section{Transfer of training from the LWJ to real world} juggling

Because the LWJ lacks the motor and haptic aspects of juggling with real balls, the goal of the third study was to evaluate the relevance and value of the skill components that can be trained in the LWJ, for the acquisition of real juggling. The main question was whether training the pattern memory and the temporalspatial relations of the 3-ball cascade, which was shown to improve control and flexibility in virtual juggling performance in the LWJ, benefits the acquisition of real juggling skills. Participants practiced the acquisition of the 3-ball cascade juggling trick for 10 days. Real balls juggling performance was measured in the highest number of consecutive successful juggling cycles during a 4 min. practice trial. Participants in the control group practiced 3BC juggling during 10 sessions each containing eight 4 minute practice trails. The experimental groups had only 5 sessions of training with real balls the other 5 were conducted in the LWJ. Juggling under speed increase was administered on session 2 and 3 and emphasis change manipulations emphasizing either height or duration on session 5, 7, session 9 was conducted with a mix of high and duration changes. Subjects in the experimental group practiced with real balls on sessions $1,4,6,8$, and 10 (the test session). On day 11 , both groups were instructed to juggle with real balls while instructed to perform tossing high or catching duration changes in specific 4 min. juggling trials

Results show that the experimental group trainees who shared their time between real ball training and the LWJ obtained comparable juggling performance levels to the control group trainees who were trained for all 10 sessions with real balls. Figure 4 depicts the two groups mean highest number of consecutive cycles of juggling real balls, over the course of 10 days. During the 11th session, trainees who were previously given LWJ training were better able to follow the instructions to change their juggling performance to cope with required toss and catch changes Figure 5 shows the mean highest number of consecutive cycles under normal conditions and when changes in catch duration and toss height were called upon.

\section{Conclusion}

We found that the audio-tactile Closed Loop mode outperformed the Open Loop solution. The information given in these two modes strongly differed. The former mode corresponds to an enactive interface in which absolute and relative timing is not prescribed, while the latter can be considered as a sort of feed forward intervention by the training system, in which timing relations are explicitly provided by the stimulation. In the second evaluation stud we examined the role the cognitive components of the juggling skill can play when directly addressed in a light weight training platform, with an attempt to understand their role and 
contribution to learning and performance of juggling. The results obtained support the value of the LWJ as a virtual reality training platform and show that subjects who received only half of their training time with real balls were not inferior in their juggling performance and were better able to apply voluntary changes in juggling format.

\section{Acknowledgements}

This work has been funded by the European Integrated Project SKILLS (FP6-IST-2005-035005-IP).

\section{References}

[1] J.R. Anderson. The architecture of cognition. Cambridge, MA: Harvard University Press, 1983.

[2] P.J. Beek, M.T. Turvey. Temporal patterning in cascade juggling. Journal of Experimental Psychology: Human Perception and Performance, 18, 934-947, 1992.

[3] P.J. Beek, A.A.M. van Santvoord. Learning the cascade juggle: A dynamical systems analysis. Journal of Motor Behavior, 24, 85-94, 1992.

[4] P.J. Beek, A. Lewbel. The science of juggling. Scientific American, 93-97, 1995.

[5] J. Cardinal, S. Kremer and S. Langerman. Juggling with Pattern Matching. Theory of Computing Systems, 39, pages 425-437, 2006.

[6] G.C. DeGuzman, J.A.S. Kelso. Multifrequency behavioral patterns and the phase attractive circle map. Biological Cybernetics, 64:485-495, 1991.

[7] B. Draganski, C. Gaser, V. Busch, G. Schuierer, U. Bogdahn, et al. Neuroplasticity: Changes in grey matter induced by training. Nature, 427: 311-312, 2004.

[8] J. Driemeyer, J. Boyke, C. Gaser, C. Büchel, A. May, Changes in Gray Matter Induced by Learning-Revisited. PLoS ONE, 3: e2669, 2008.

[9] R. Ehrenborg, M. Readdy. Juggling and applications to qanalogues. Discrete Math., 157:107-125, 1996.

[10] P.W. Fink, J.A.S. Kelso, P. Foo, V.K. Jirsa. Local and global stabilization of coordination by sensory information. Experimental Brain Research, 134:9-20, 2000.

[11] D. Gopher. Control processes in the formation of task units. In: Qicheng Jing (Ed.): Psychological Science around the World, Volume 2, Social and Applied Issues. Oxford Psychology Press. A chapter based on a keynote address given at the 28th International Congress

of Psychology, 2006.

[12] D. Gopher. Emphasis change as a training protocol for high demands tasks. In: A. Kramer, D. Wiegman, A. Kirlik (Eds): Attention: From Theory to Practice. Oxford Psychology Press, 2007.

[13] P.S. Haibach, G.L. Daniels, K.M. Newell. Coordination changes in the early stages of learning to cascade juggle. Human Movement Science, 23, 185-206, 2004.

[14] H. Haken, C.E. Peper, P.J. Beek, A. Daffertshofer. A model for phase transitions in human hand movements during multifrequency tapping. Physica D, 90:179-196, 1996.

[15] R. Huys, A. Daffertshofer, P.J. Beek. Multiple time scales and multiform dynamics in learning to juggle. Motor Control, 8, 188-212, 2004.

[16] V.K. Jirsa, P.W. Fink, P. Foo, J.A.S. Kelso. Parametric stabilization of biological coordination: a theoretical model. Journal of Biological Physics, 26:85-112, 2000.

[17] J.A.S. Kelso, G.C. DeGuzman. Order in time: how cooperation between the hands informs the design of the brain. In: Haken $\mathrm{H}$ (ed) Neural and synergetic computers. Springer, Berlin Heidelberg New York, pp 180-196, 1988.

[18] J. Lagarde, J.A.S. Kelso. The binding of movement, sound and touch: Multimodal coordination dynamics. Experimental Brain Research, 173, 673-88, 2006.

[19] G.D. Logan. Toward an Instance Theory of Automatization, Psychological Review, 492-527, 1988.

[20] C.E. Peper, P.J. Beek, P.C.W. Van Wieringen. Coupling strength in tapping a 2:3 polyrhythm, Human Movement Science Volume, 14, 217-245, 1995.

[21] A.A. Post, A. Daffertshofer, P.J. Beek. Principal components in three-ball cascade juggling. Biological Cybernetics, 82, 143-152, 2000.

[22] B. Tuller, J.A.S. Kelso. Environmentally specified patterns of movement coordination in normal and split-brain subjects. Experimental Brain Research, 75:306-316, 1989.

[23] P.G. Zanone, J.A.S. Kelso. Evolution of behavioral attractors with learning: Nonequilibrium phase transitions. Journal of Experimental Psychology: Human Perception and Performance, 18, 403-421, 1992. 